\title{
TEMA 14-2014: MANEJO DE LA ASGITIS MALIGNA NO REFRACTARIA
}
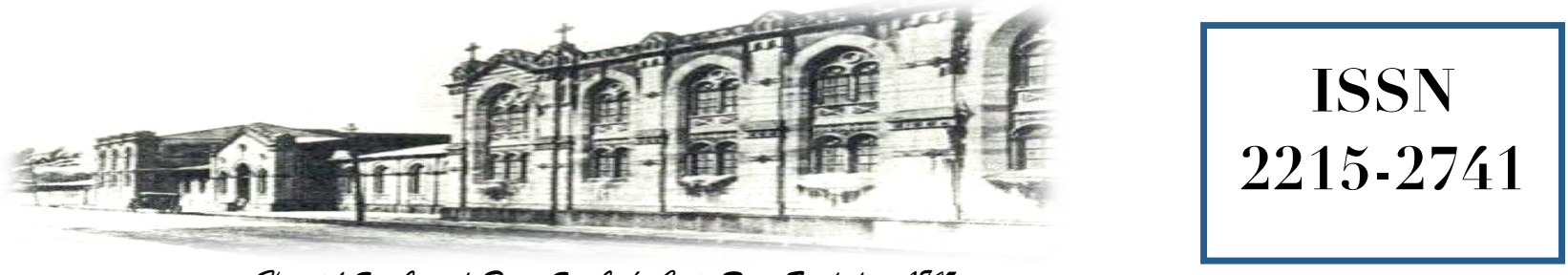

Haspital San quan de Dias. San Jasé, Casta Rica. Fundada en 1845

Recibido: $\quad 27 / 02 / 2014$

Aceptado: $\quad 24 / 04 / 2014$

Andrea M. Medina Méndez ${ }^{1}$

David F. Ávalos Chacón ${ }^{2}$

${ }^{1}$ Residente de Medicina Paliativa de Adultos del PPEM. UCR-CENDEISSS. Caja Costarricense de Seguro Social. Costa Rica. Correo electrónico: andreamedinacr@hotmail.com

${ }^{2}$ Geriatra y Médico Paliativa del Centro Nacional de Control del Dolor y Cuidados Paliativos. Caja Costarricense del Seguro Social. Director del postgrado de Medicina Paliativa. UCR-CENDEISSS. Costa Rica.

\section{RESUMEN}

Las AM es una complicación que con frecuencia se encuentra en la fase terminal de varias neoplasias, sobre todo en las intraabdominales como el cáncer de ovario, colorectal, páncreas y útero. Sin embargo su manejo difiere de la ascitis por otra causa ya que su fisiopatología es muy diferente, aunque pueden coincidir en algunos aspectos como en el uso de diuréticos. Para lograr un mejor manejo de la AM, su líquido debe de ser estudiado y clasificado, ya que será ésta clasificación la que dará la pauta a seguir con respecto al tratamiento a brindar: diuréticos, paracentesis, catéteres y derivaciones. Ante el aumento importante que se prevé de neoplasias terminales para las próximas décadas a nivel mundial, hay que familiarizarse con abordaje adecuado de la AM.

\section{PALABRAS CLAVE}

Ascitis maligna. Estadio terminal. Tratamiento.

\begin{abstract}
The malignant ascites (MA) is a complication that frequently is in the terminal stage of various malignancies, especially abdominal as ovarian, colorectal, pancreas and uterus. However, handling differs from other causes of ascites since its pathophysiology is different although, some aspects may overlap as in the use of diuretics. For better management of the MA, the peritoneal liquid must be studied and classified, because this classification will be the leading feature to providing treatment: diuretics, paracentesis, catheters and shunts. Given the expected significant increase of end stage neoplasms for the next decades in the word, we must be familiar with the proper approach of MA.
\end{abstract}

\section{KEY WORDS}

Malignant ascites. Terminal stage. Treatment. 


\section{ABREVIATURAS}

AM: Ascitis maligna.

MMP: Matriz metaloproteinasa.

SAAG: Gradiente albúmina sérica-albúmina ascítica.

SRAA: Sistema renina-angiotensina-aldosterona VEGF: Factor de Crecimiento Vascular Endotelial.

VPF: Factor de Permeabilidad Vascular.

TIPS: Cortocircuito portosistémico intrahépatico transyugular.

\section{DISCUSIÓN}

La palabra ascitis viene del griego askos que significa saco/ bolsa ${ }^{(1)}$. Es el término empleado para denotar la efusión de líquido en la cavidad peritoneal y cuando ese líquido está asociado a un padecimiento oncológico se habla de una $\mathrm{AM}^{(2,3)}$. El Instituto Nacional del Cáncer define la AM como una acumulación de fluido que contiene las células cancerosas en el abdomen $^{(4,5)}$. La Organización Mundial de la Salud (OMS) prevé que las muertes por cáncer sigan aumentando en todo el mundo y que lleguen a alcanzar cifras de 13.1 millones de defunciones para el $2030^{(6)}$; nuestro país no será la excepción de este aumento.

La AM representa el $10 \%$ de todas las ascitis $^{(1,5,7,8-11)}$. La esperanza de vida a partir del diagnóstico de AM es de 20 semanas $^{(11)}$, aunque para otros autores el tiempo puede ser variable (2 a 6 meses según Saiz). Por otro lado, Sales refiere que la supervivencia media desde el momento de la realización de la primera paracentesis en pacientes con AM es de aproximadamente 2 meses. La calidad de vida puede mejorar con los procedimientos paliativos y no existe una terapia anti-tumoral efectiva ${ }^{(11)}$.

La AM se puede presentar como primer síntoma de una enfermedad oncológica en el 52 a $54 \%$ de los $\operatorname{casos}^{(1,11)}$ y de esos un $20 \%$ tienen un tumor primario desconocido ${ }^{(4,7,9,12)}$.

Para Markman ${ }^{(13)}$ esta condición es relativamente común en la historia natural de los pacientes con cáncer de ovario y del tracto gastrointestinal. Puede causar una considerable angustia física y puede afectar muy negativamente la capacidad funcional de una persona durante los meses restantes de su vida.
Las neoplasias intraabdominales que más comúnmente causan la AM son: ovario, colorectales, páncreas y útero. Dentro de las extraabdominales: linfomas, pulmón y mama ${ }^{(11)}$.

Sin embargo no existen protocolos (nacionales ni internacionales) claros en relación al manejo de esta patología, que se espera vaya en aumento junto al aumento de la incidencia de cánceres que se tiene proyectado. Por lo que se procedió a realizar una búsqueda en las diferentes revistas internacionales para así llegar a un consenso final y definir el mejor modo de abordar esta patología

\section{Fisiología del peritoneo sano}

El peritoneo está compuesto de tejido mesotelial y contiene un aproximado de $100 \mathrm{ml}$ de líquido peritoneal libre, para lubricar la serosa. La presencia de este líquido va a estar influenciado por la presión portal, la presión oncótica, el equilibrio agua-sodio y la permeabilidad de los canales vasculares a los macronutrientes y células.

Normalmente los capilares peritoneales filtran el plasma e intercambian sustancias y células. Este filtrado pasa a través de las "foraminas" (estromas/orificios) a lo largo del epitelio escamoso del peritoneo parietal y de éste es drenado por los canales linfáticos ("stomata") hasta la capa de serosa. Finalmente, llega a la vena subclavia a través de los estromas mediastinales y el conducto torácico derecho (que drena gracias a la presión intratorácica negativa). Aproximadamente $2 / 3$ del líquido son reabsorbidos por los canales linfáticos y distribuidos bajo el diafragma ${ }^{(14)}$.

\section{Fisiopatología de la ascitis maligna}

Es compleja, multifactorial e involucra tanto la producción como la reabsorción del líquido ascítico. La fisiopatología de la AM es diferente a la de causas no oncológicas.

La neovascularización provoca un aumento de la permeabilidad capilar, seguida por una obstrucción en el drenaje (oclusión peritoneal linfática "stomata"). Por otro lado también hay un aumento de la permeabilidad de los vasos tumorales y producción forzada de líquido peritoneal. La carcinomatosis a su vez provoca una neovascularización del peritoneo parietal y aumenta la pro- 
ducción de glicoproteínas, que a su vez crean un aumento en la permeabilidad vascular de los pequeños vasos sanguíneos ${ }^{(14)}$.

El VEGF altera la permeabilidad vascular y es el responsable del crecimiento vascular incontrolado de la masa tumoral. El VEGF se encuentra en concentraciones muy altas en los cánceres de origen gástrico, colorectal y ovario. También se une al receptor del VPF que posteriormente activa la tirosina quinasa. Este mecanismo explica la eficacia de las terapias que inhiben la interacción entre VEGF y su receptor.

Dado que la ascitis de muchos pacientes con malignidad tiene un alto contenido de proteínas, se ha propuesto que hay una alteración en la permeabilidad vascular, como factor implicado en la patogénesis de la $\operatorname{ascitis}^{(7)}$.

Debido a la disminución de la eliminación de líquido como consecuencia de la obstrucción de vasos linfáticos, el volumen de sangre circulante se reduce y esto activa el SRAA, que conduce a la retención de sodio $^{(7)}$.

\section{Otras hipótesis}

Papel de las proteínas de la MMP: la gelatinasa y la estromelinasa-3 son las principales y se encargan de romper la matriz durante la invasión cancerígena y también generan un aumento en la permeabilidad vascular. La inflamación peritumoral también aumenta la permeabilidad vascular.

Mecanismo hormonal: el aumento de la ascitis causa una disminución en el volumen circulatorio sanguíneo que, como consecuencia, activa el SRAA y así la retención de sodio.

Se dice que el 50\% de los casos de AM son causados por invasión del peritoneo parietal o visceral, en un $15 \%$ por invasión hepática o por compresión de la vena porta, un $20 \%$ por invasión linfática y hasta un $15 \%$ por una combinación de las causas anteriores ${ }^{(11)}$.

\section{Cuadro clínico}

Es muy variable y el predominio de un síntoma $u$ otro y la severidad del mismo depende del aumento de la presión intraperitoneal.
Puede encontrarse: disnea, náuseas, anorexia, fatiga, reflujo gastroesofágico, distención abdominal, dolor abdominal, limitación a la movilidad, distress respiratorio, anasarca, saciedad temprana, aumento de peso, cambios en los hábitos intestinales y edema periférico, escrotal y peneano.

Dentro del examen físico se puede mencionar la presencia de abombamiento de los flancos (cuando hay entre 500 y $1000 \mathrm{ml}$ de líquido), matidez en los flancos, el signo de la ola, el signo del charco (cuando hay entre 300 y $400 \mathrm{ml}$ de líquido), hernia abdominal, matidez a la percusión de la base del hemitoráx derecho (derrame pleural) y edemas en miembros inferiores, genitales y abdomen bajo.

\section{Estudios complementarios}

Dentro de los estudios complementarios que se pueden realizar se encuentran:

1. Paracentesis diagnóstica o evacuadora

2. Estudios del líquido ascítico

3. Ultrasonido de abdomen

4. TAC de abdomen

5. Marcadores tumorales

Tanto para su diagnóstico así como para la colocación de algún tipo de catéter

\section{Análisis del líquido ascítico}

Es importante que cuando la paracentesis se realiza con fines diagnósticos, se realice un adecuado análisis del líquido obtenido, para asegurarse una clasificación adecuada y por ende un tratamiento apropiado.

Según la cantidad de proteínas dentro de ese líquido, se puede clasificar como trasudado o exudado. Donde un líquido exudado va a ser aquel que tenga:

- $\quad$ Proteínas $>2.5 \mathrm{~g} / \mathrm{dl}$

- Células sanguíneas $>250 / \mu 1$

- $\mathrm{DHL}>$ al $50 \%$ en relación con su valor sérico

\section{$S A A G$}

Se fundamenta en que la presión portal es directamente proporcional al gradiente de presión 
oncótica entre los capilares esplácnicos y la ascitis, según Salinas. Es la diferencia entre la concentración de albúmina sérica y de la albúmina del líquido ascítico. Si es $\geq 1.1 \mathrm{~g} / \mathrm{dl}$ es alta (ascitis trasudativa) y si $\leq 1.1 \mathrm{~g} / \mathrm{dl}$ es baja (ascitis exudativa). Un SAAG $\geq 1.1 \mathrm{~g} / 1$ predice una ascitis como hipertensión portal, con una precisión de aproximadamente del $97 \%{ }^{(15)}$.

"La precisión del gradiente puede verse afectada por un excesivo retraso en la extracción de la muestra de sangre respecto a la del líquido ascítico y en la ascitis quilosa debido a que los lípidos interfieren con la técnica de determinación de la albúmina"(16).

Cuadro 1. Ejemplos de patologías y el tipo de SAAG característico

\begin{tabular}{|c|c|}
\hline ALTO & BAJO \\
\hline $\begin{array}{ll}\text { Enfermedad } & \text { hepática } \\
\text { veno-oclusiva } & \end{array}$ & \multirow{2}{*}{ Inflamación peritoneal } \\
\hline $\begin{array}{l}\text { Obstrucción de la vena } \\
\text { hepática }\end{array}$ & \\
\hline $\begin{array}{l}\text { Enfermedad hepática } \\
\text { crónica }\end{array}$ & \multirow[t]{2}{*}{ Síndrome nefrótico } \\
\hline Falla hepática crónica & \\
\hline Trombosis venosa portal & \\
\hline $\begin{array}{l}\text { Hemodiálisis con sobre- } \\
\text { carga hídrica }\end{array}$ & Enfermedades crónicas \\
\hline $\begin{array}{l}\text { Metástasis hepáticas } \\
\text { masivas }\end{array}$ & \multirow{2}{*}{$\begin{array}{l}\text { Enfermedades de vísce- } \\
\text { ras abdominales (pan- } \\
\text { creatitis, biliar, quilosa, } \\
\text { carcinomatosis perito- } \\
\text { neal, infarto u obst } \\
\text { intestinal) }\end{array}$} \\
\hline Mixedema & \\
\hline
\end{tabular}

Otras pruebas y análisis que pueden realizarse al líquido ascítico, con su respectiva interpretación clínica son las siguientes:

- Aumento de la amilasa: ascitis pancreática

- Aumento de los triglicéridos: ascitis quilosa

- Leucocitos $>350 / \mathrm{mm}^{3} \rightarrow$ infección

- PMN: sospechar infección bacteriana

- Mononucleares: infección micótica o TB

- Eritrocitos $>50.000 / \mu \mathrm{l}$ : ascitis hemorrágica, malignidad, tuberculosis o secundaria a trauma.

- $\mathrm{pH}<7$ sugiere infección bacteriana

- Citología: es considerada el patrón de oro para el diagnóstico ${ }^{(1,11)}$ es positiva por lesiones malignas solo entre 50 y $60 \%$ de los ca$\operatorname{sos}^{(11)}$; pero hay autores que dicen que este valor se eleva hasta el $90 \%$ cuando hay carcinomatosis peritoneal $^{(11,16)}$.

- Glucosa: < $60 \mathrm{mg} / \mathrm{dl} \rightarrow$ carcinomatosis.

- Cultivo y tinción de Gram: confirmar el diagnóstico de infección bacteriana

- Marcadores tumorales ${ }^{(11)}$ : antígenocarcinoembrionario, $\alpha$ fetoproteína; Ca 125

- La inmunohistoquímica no es útil.

Cuando se documenta una citología positiva por malignidad en un paciente no conocido portador de una neoplasia, se debe realizar los estudios correspondientes en busca del tumor primario. En los pacientes masculinos con citología positiva, cuyos estudios son negativos pese a los análisis de sangre y obtención de imágenes radiológicas, puede no ser útil más estudios, dado que no va a afectar a la gestión o el resultado de la enfermedad $^{(11)}$.

Cuadro 2. Principal sospecha diagnóstica según las características del líquido ascítico.

\begin{tabular}{|c|c|c|}
\hline Estudio & Hallazgos & Diagnóstico \\
\hline Proteínas & $\begin{array}{l}<2,5 \mathrm{~g} / \mathrm{dl} \\
>2,5 \mathrm{~g} / \mathrm{dl}\end{array}$ & $\begin{array}{l}\text { Trasudado } \\
\text { Exudado }\end{array}$ \\
\hline & $>1,1 \mathrm{~g} / \mathrm{dl}$ & $\begin{array}{l}\text { Ascitis asociada a } \\
\text { hipertensión portal }\end{array}$ \\
\hline SAAG & $<1,1 \mathrm{~g} / \mathrm{dl}$ & $\begin{array}{l}\text { Ascitis NO asociada a } \\
\text { hipertensión portal }\end{array}$ \\
\hline Triglicéridos & $>110 \mathrm{mg} / \mathrm{dl}$ & Ascitis quilosa \\
\hline Leucocitos & $\mathrm{PMN}>250$ & $\begin{array}{l}\text { Peritonitis bacteriana } \\
\text { espontánea }\end{array}$ \\
\hline por $\mathrm{mm}^{3}$ & $\begin{array}{l}\text { Linfocitos } \\
>1.000\end{array}$ & Peritonitis tuberculosa \\
\hline $\begin{array}{l}\text { Hematíes por } \\
\mathrm{mm}^{3}\end{array}$ & $>50.000 / \mathrm{mm}^{3}$ & $\begin{array}{l}\text { Ascitis hemorrágica: } \\
\text { sugestiva de carcino- } \\
\text { matosis peritoneal, } \\
\text { ascitis pancreática, } \\
\text { hepatocarcinoma con } \\
\text { diseminación a cavi- } \\
\text { dad peritoneal, ascitis } \\
\text { tuberculosa }\end{array}$ \\
\hline Citología & Positivo & $\begin{array}{l}\text { Carcinomatosis peri- } \\
\text { toneal }\end{array}$ \\
\hline $\begin{array}{l}\text { Cultivo } \\
\text { microbiológico }\end{array}$ & Positivo & $\begin{array}{l}\text { Peritonitis. Falsos } \\
\text { negativos en el } 30 \%\end{array}$ \\
\hline
\end{tabular}

Sin embargo, en pacientes de sexo femenino, si los métodos convencionales no han podido demostrar que el tumor de origen, la laparoscopia o laparotomía se debe realizar. Porque las pacientes con una neoplasia de ovario son sensibles a citorreducción tumoral y quimioterapia y los 
resultados en cuanto a supervivencia son mejores $^{(11)}$.

\section{Clasificación de la ascitis maligna}

La AM se puede clasificar de diferentes maneras:

1. Según el aclaramiento del líquido y el SAAG (cuadro 3):

Cuadro 3 Clasificación de la AM según el aclaramiento de líquido y el SAAG.

\begin{tabular}{|l|l|l|l|}
\hline \multicolumn{2}{|l|}{ Aclaramiento y SAAG $>1.1$} & $\begin{array}{l}\text { Aclaramiento } \\
\text { y SAAG }<1.1\end{array}$ & Quiloso \\
\hline Central & Mixto & Periférica & $\begin{array}{l}\text { Obstrucción } \\
\text { linfática }\end{array}$ \\
\hline $\begin{array}{l}\text { Afectación } \\
\text { intrahepática }\end{array}$ & $\begin{array}{l}\text { Afectación } \\
\text { intrahepática } \\
\text { o peritoneal }\end{array}$ & $\begin{array}{l}\text { Afectación } \\
\text { peritoneal }\end{array}$ & $\begin{array}{l}\text { Frecuentemente } \\
\text { retroperitoneal }\end{array}$ \\
\hline
\end{tabular}

Ascitis Central: El tumor invade el parénquima hepático y provoca una compresión de los sistemas linfáticos y/o venoso portal, por ende hay una disminución de la presión oncótica como resultado de la ingesta limitada de proteínas y el estado catabólico

Ascitis Periférica: Hay depósitos de células tumorales en la superficie del peritoneo parietal y visceral que, interfieren con la mecánica del drenaje venoso y/o linfático. El bloqueo está a nivel del espacio peritoneal más que a nivel del parénquima hepático.

Ascitis Mixta: El tumor está presente tanto a nivel hepático como en la superficie peritoneal.

Ascitis Quilosa: Hay infiltración tumoral del espacio retroperitoneal que provoca obstrucción del fluido a través de los nódulos linfáticos y/o el páncreas.

2. Según su severidad (cuadro 4):

Cuadro 4. Clasificación según severidad

\begin{tabular}{|c|c|c|}
\hline $\begin{array}{l}\text { Grado } 1^{\circ} \\
\text { Leve }\end{array}$ & $\begin{array}{l}\text { Grado } 2^{\circ} \\
\text { Moderada }\end{array}$ & $\begin{array}{l}\text { Grado } 3^{\circ} \\
\text { Severa }\end{array}$ \\
\hline $\begin{array}{l}\text { La ascitis solo se } \\
\text { puede diagnosti- } \\
\text { car por ultrasoni- } \\
\text { do }\end{array}$ & $\begin{array}{l}\text { Hay disten- } \\
\text { ción abdo- } \\
\text { minal sensi- } \\
\text { ble }\end{array}$ & $\begin{array}{l}\text { Distensión mar- } \\
\text { cada o distensión } \\
\text { abdominal a } \\
\text { tensión }\end{array}$ \\
\hline
\end{tabular}

3. Según la presencia de complicaciones (cuadro 5):

Cuadro 5. Clasificación de la AM según complicación.

\begin{tabular}{|l|l|}
\hline No complicada & Complicada \\
\hline $\begin{array}{l}\text { Ascitis SIN infección y } \\
\text { sin síndrome hepato- } \\
\text { renal }\end{array}$ & $\begin{array}{l}\text { Ascitis CON infección } \\
\text { y/o síndrome hepato- } \\
\text { renal }\end{array}$ \\
\hline
\end{tabular}

4. Según presencia de refractariedad (cuadro 6):

Cuadro 6 Clasificación de la AM según la refractariedad

\begin{tabular}{|ll|}
\hline \multicolumn{2}{|c|}{ Criterios de Refractariedad } \\
\hline Resistente a diuréticos & Intratable con diuréticos \\
\hline Ascitis que no responde & El uso de diuréticos indu- \\
a la restricción de sodio & ce a efectos adversos \\
dietético y a altas dosis & limita el uso del diurético \\
de diuréticos & a dosis efectivas \\
\hline
\end{tabular}

Fuente: Med Clin N Am 93 (2009) 801-817

\section{Abordaje terapéutico}

La selección de una modalidad de tratamiento apropiado sigue siendo un cuidadoso proceso, que debería tener en cuenta:

- El riesgo potencial

- Los beneficios

- La esperanza de vida del paciente según su pronóstico

Dentro de los factores de pobre pronóstico se encuentra la presencia de edema periférico, la hipoalbuminemia importante y la presencia de metástasis hepáticas; mientras que la supervivencia prolongada se encontró en los pacientes con cáncer de ovario ${ }^{(11)}$.

El manejo de la AM también depende de su fisiopatología y del estado global del paciente y está encaminado a la paliación de síntomas, con excepción del cáncer de ovario, que se trata con cirugía citoreductiva y quimioterapia ${ }^{(18)}$.

En relación al manejo médico de la AM lo primero que se debe hacer es clasificarla según el SAAG y con base en esta clasificación iniciar el manejo (algoritmo 1) 
Figura I. Algoritmo propuesto para el abordaje de la ascitis maligna

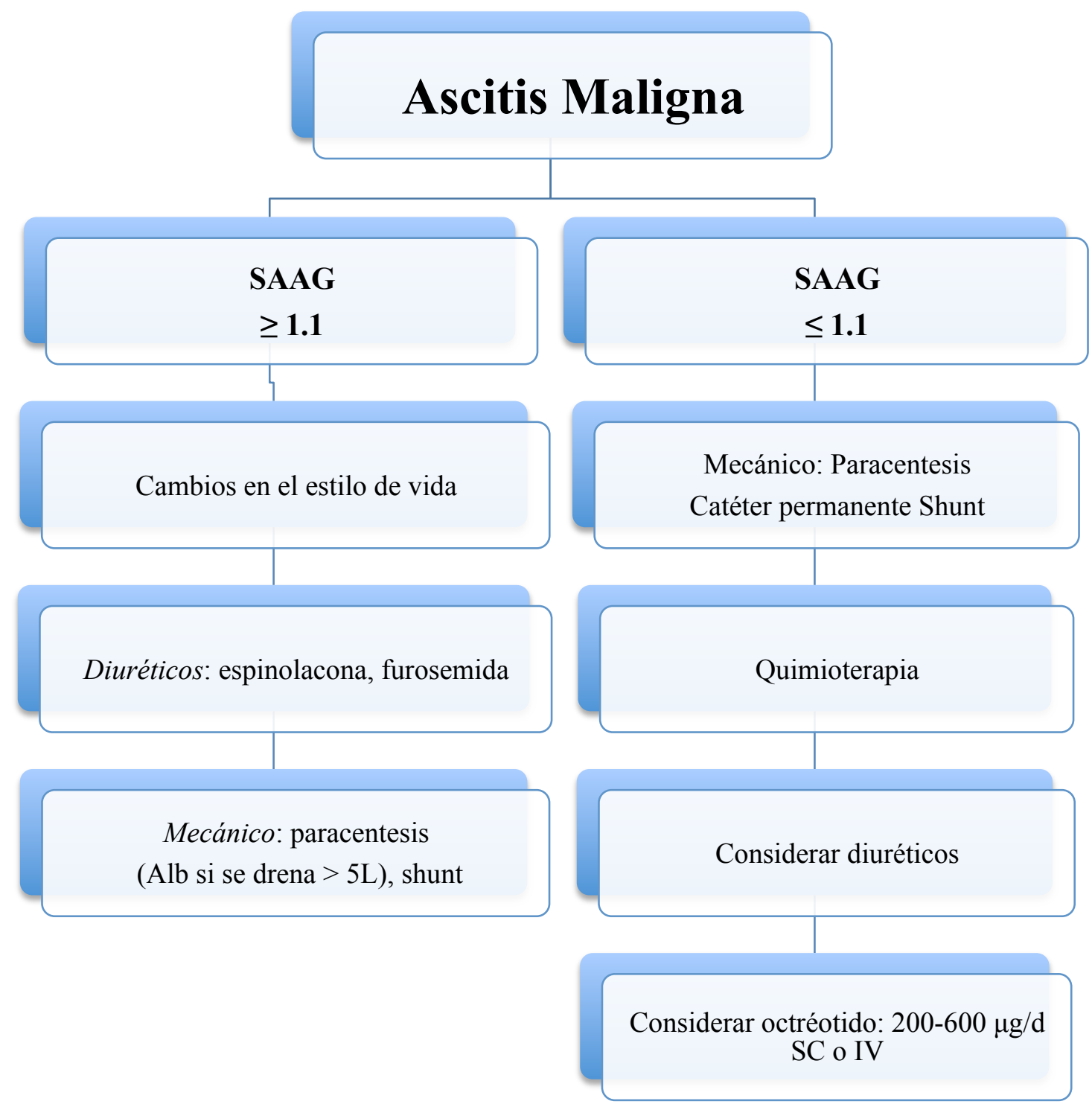

Fuente Tarascon. Palliative Medicine Pocketbook. 2012. Pag 52 (20)

Si el paciente tiene un $\mathrm{SAAG} \geq 1.1$; se considera que hay hipertensión portal y lo primero que se debe realizar son cambios en el estilo de vida, restringiendo la ingesta de sodio y líquidos, sin embargo, se considera que los cambios en el estilo de vida pueden ser muy tediosos para los pacientes en condiciones de terminalidad.

Si los cambios en el estilo de vida no son efectivos, ya sea por falta de apego o por falta de respuesta a pesar del apego, se puede iniciar el tratamiento con diuréticos ahorradores de potasio o diuréticos de asa.
Se inicia con la espironolactona, hasta llegar a un máximo de $400 \mathrm{mg} / \mathrm{d}$. Toma 2 semanas ver el efecto completo del diurético.

Si los cambios en el estilo de vida y el uso de diuréticos falla, se debe realizar una paracentesis. Si se drena más de 5 litros; se debe valorar reponer 6 a $8 \mathrm{~g}$ de albúmina endovenosa por cada litro removido. Se recomienda utilizar albúmina al $5 \%$ si hay hipovolemia y al $25 \%$ si hay hipervolemia. Por último, se puede considerar el uso de TIPS (Cortocircuito portosistémico intrahepático transyugular) en pacientes con pronóstico limitado a corto plazo. 
Por lo contrario, si el SAAG es $\leq 1.1$, se considera que no hay hipertensión portal. Aquí la primera opción de tratamiento es la remoción mecánica, ya sea con paracentesis de alto volumen, sin compromiso hemodinámico o con catéteres permanentes, para que el paciente se autodrene en la casa pequeños volúmenes. También se puede utilizar las derivaciones peritoneo-venosas (derivación de LeVeen o Denver) pero debe reservarse a pacientes con pronóstico de vida menor a 3 meses o con ascitis refractaria. Se considera de gran utilidad en pacientes con cáncer de ovario con función renal normal.

También se puede considerar el uso de quimioterapia sistémica o intraperitoneal. Se puede hacer una prueba terapéutica con diuréticos.

\section{Uso de Diuréticos (cuadro 7)}

Es considerada por algunos autores la primera línea de tratamiento. No hay estudios randomizados y controlados sobre la eficacia real en reducir la ascitis y los síntomas. Se considera efectiva en el $40 \%$ pacientes con $\mathrm{AM}^{(8)}$. Un estudio fase II sugirió que la eficacia de los diuréticos en AM depende de la concentración plasmática de renina/aldosterona. No hay ningún método para predecir cuál paciente con AM va a responder a los diuréticos ${ }^{(7)}$.

Con los diuréticos se logra reabsorber un máximo de $800 \mathrm{ml}$ por día, con una pérdida de $1 \mathrm{~kg} /$ día. Su eficacia es mayor cuando la ascitis es secundaria a metástasis hepáticas con hipertensión portal e hipoalbuminemia ${ }^{(14)}$.

Las dosis máximas recomendadas son

- Espironolactona: $400 \mathrm{mg} / \mathrm{d}$

- Furosemida: $160 \mathrm{mg} / \mathrm{d}$

\section{Paracentesis}

Es la terapia más común y efectiva. Logra un alivio temporal de los síntomas en el $90 \%$ de los $\operatorname{casos}^{(5)}$. Se considera un procedimiento seguro y tiende a mostrar una eficacia reducida con el paso del tiempo ${ }^{(9)}$.

Los principales riesgos son el daño visceral, el sangrado, desarrollo de sepsis, hipotensión arterial, daño renal y fuga de líquido.
Cuadro 7. Consideraciones generales del uso de diuréticos en el tratamiento de la AM.

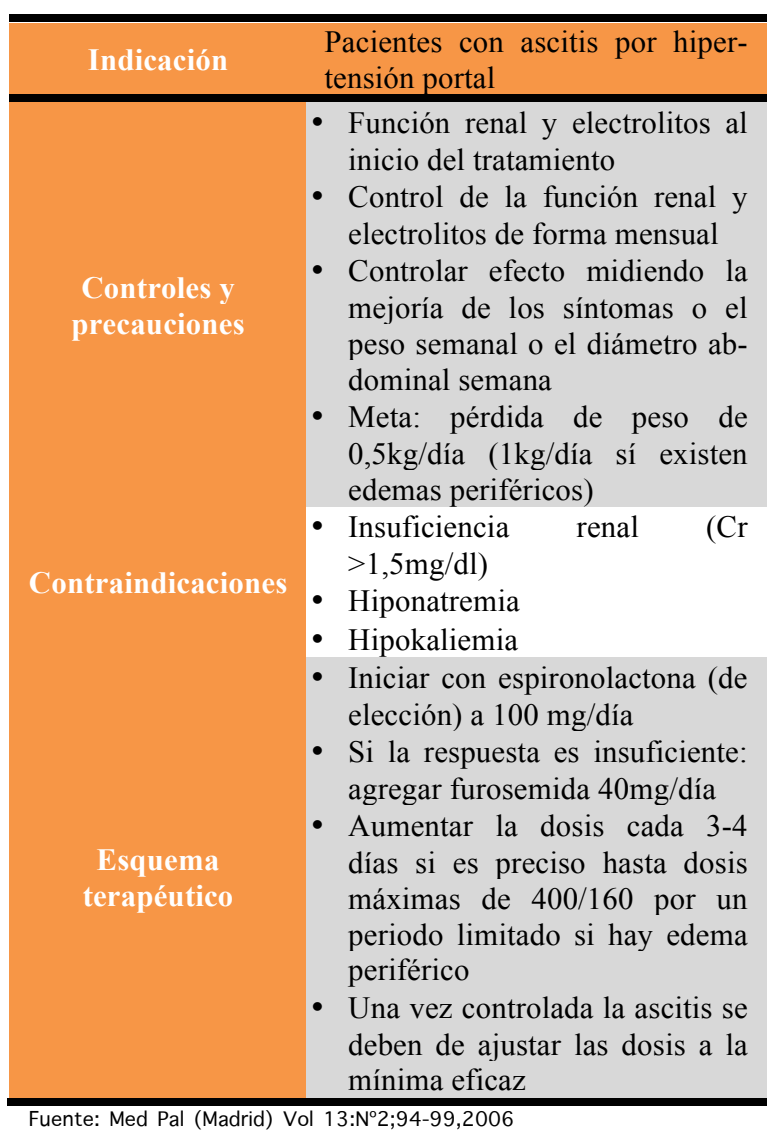

Dentro de las ventajas del procedimiento se encuentran que es rápido, simple, de bajo costo $\mathrm{y}$ con un inminente alivio de los síntomas (temporal).

Las complicaciones de la paracentesis dependen del mecanismo causal de la ascitis. En pacientes con HP son las mismas que en los pacientes cirróticos. La principal es el síndrome de disfunción hemodinámica post paracentesis (hipotensión, activación del sistema antinatriurético y fracaso renal). Otras complicaciones, por orden de frecuencia son la hiponatremia, el deterioro renal, la encefalopatía hepática, el sangrado digestivo y la infección bacteriana secundaria ${ }^{(16)}$.

No hay estudios randomizados controlados que hayan evaluado la seguridad y eficacia de las paracentesis en $\mathrm{AM}^{(7)}$.

Las paracentesis repetidas, de grandes volúmenes sin expansión del volumen plasmático pueden 
estar asociadas con un número significativamente mayor de episodios de hipotensión e insuficiencia renal $^{(7)}$.

No hay estudios randomizados comparativos entre los diuréticos y la paracentesis en la ascitis maligna $^{(7,14)}$. Los estudios aleatorizados no mostraron diferencias significativas en la supervivencia entre los pacientes tratados con albúmina y los tratados con otros expansores del plasma ${ }^{(7)}$.

La severidad de los síntomas se correlaciona con la presión intraperitoneal previa al procedimiento pero no con el volumen de líquido de ascitis ${ }^{(17)}$. Antes de su realización se debe valorar el riesgo del paciente para el desarrollo de complicaciones (cuadro 8) y es difícil predecir qué pacientes pueden presentar estas complicaciones ${ }^{(16)}$.

Hay autores que consideran que la albúmina sérica baja (cuadro 9), puede ser un factor pronóstico independiente; principalmente en las neoplasias diferentes al cáncer de ovárico. También se incluyen las metástasis hepáticas y la elevación de la bilirrubina sérica. Un líquido ascítico alto en proteínas y hemorrágico tiene mayor riesgo de obstrucción del catéter ${ }^{(2)}$.
Cuadro 8. Factores de riesgo para el desarrollo de complicaciones con la paracentesis

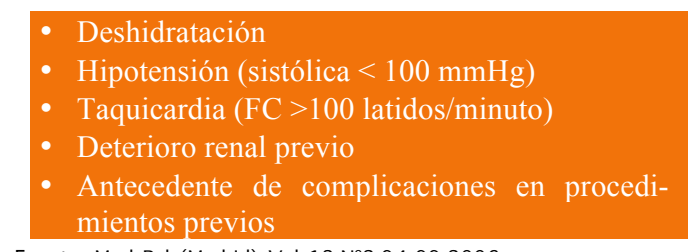

Fuente: Med Pal (Madrid) Vol 13:N²;94-99,2006

El drenaje repetido de grandes volúmenes de ascitis se ha asociado con reducciones significativas de los niveles de proteínas séricas ${ }^{(9,10)}$.

El alivio de los síntomas con la parecentesis suele permanecer durante un promedio de 10 días ${ }^{(17)}$. En los pacientes que precisan paracentesis de repetición ( $>1$ cada 7 a 15 días) debería plantearse el uso de catéteres permanentes ${ }^{(16)}$.

Coupe $^{(19)}$ es su estudio encontró que los puertos peritoneales son eficaces, seguros y están asociados con un alivio sintomático en la mayoría de los pacientes.

\begin{tabular}{|c|c|c|c|}
\hline $\begin{array}{l}\text { Características } \\
\text { del paciente }\end{array}$ & $\begin{array}{l}\text { Factores de } \\
\text { riesgo }\end{array}$ & Técnica & Consideraciones \\
\hline Con HP asociada & No & $\begin{array}{l}\text { Drenaje } \\
\text { rápido } 51 \text { en } \\
2 \mathrm{a} 4 \mathrm{~h}\end{array}$ & $\begin{array}{l}\text { Infusión de } 1 \text { vial de albúmina al } 20 \% \text { por c/ } 1.51 \text { de drenaje. } \\
\text { Inicio de la infusión simultáneamente con el drenaje }\end{array}$ \\
\hline Con HP asociada & $\mathrm{Si}$ & $\begin{array}{l}\text { Drenaje lento } \\
1.51 \text { por h }\end{array}$ & $\begin{array}{l}\text { Infusión de } 1 \text { vial de albúmina al } 20 \% \text { por c/ } 1.51 \text { de drenaje. } \\
\text { Inicio de la infusión simultáneamente con el drenaje }\end{array}$ \\
\hline Sin HP asociada & No & $\begin{array}{l}\text { Drenaje } \\
\text { rápido } 51 \text { en } \\
2 \mathrm{a} 4 \mathrm{~h}\end{array}$ & $\begin{array}{l}\text { No necesita albúmina. Monitorizar presión arterial (PA). } \\
\text { Si PA es }<100 \mathrm{mg} / \mathrm{Hg} \text { o FC }>100 \text { por minuto; detener el drenaje } \\
\text { e iniciar perfusión con solución salina }\end{array}$ \\
\hline Sin HP asociada & $\mathrm{Si}$ & $\begin{array}{l}\text { Drenaje lento } \\
1.51 \text { en por } h\end{array}$ & $\begin{array}{l}\text { No necesita albúmina. Monitorizar PA. } \\
\text { Si PA es }<100 \mathrm{mg} / \mathrm{Hg} \text { o FC }>100 \text { por minuto; detener el drenaje } \\
\text { e iniciar perfusión con solución salina }\end{array}$ \\
\hline
\end{tabular}

Nota: en todos los casos se considera un volumen máximo de drenaje de 51 por sesión.

Fuente: Med Pal (Madrid) Vol 13:№2;94-99,2006

\section{Catéteres}

Aquellos pacientes a los que hay que realizarles paracentesis frecuentes ( $<7$ días) son los que ven más beneficiados. El drenaje puede ser permanente o intermitente para así evitar la tensión. Pueden permanecer meses en su lugar y la sobrevida con el catéter va de 15 días a 18 meses.
Beneficios: autodrenaje, elimina la necesidad de hospitalización y menor disconfort.

Complicaciones: peritonitis (la frecuencia de aparición es muy variable), celulitis, fuga en el sitio de punción, infección del catéter, oclusión del catéter (ser produce entre un 15 y un $30 \%$ de los casos), fuga del catéter e hipotensión severa (el riesgo de hipotensión arterial es de un 5\% 
aproximadamente). No se ha descrito situaciones de perforación intestinal.

Contraindicaciones: síndrome adherencial, carcinomatosis y líquido loculado

Existen muchas opciones de catéteres: "pig tail" pleurX, Tenckhoff, Port-A-Cath y de diálisis (tunelizados). Su colocación se puede realizar previa coordinación con el servicio de radiología. El tipo de catéter que se coloque dependerá del que disponga cada servicio.

La mayoría de los autores prefieren los catéteres tunelizados porque se asocian con un menor riesgo de infección y tienen mayor estabilidad que los "pig-tail" estándares.

La infección del catéter está más asociada con la duración, por lo que debe usarse en pacientes con expectativa de vida de semanas y no meses. Se entrena al paciente para que se drene a diario por las primeras 2 semanas y luego $\mathrm{PRN}^{(9)}$.

Saiz et al. estudiaron la seguridad y efectividad de los catéteres tunelizados permanentes en la AM. Los catéteres se mantuvieron permeables una mediana de 52 días en los 8 de 10 pacientes que fallecieron. Sólo un catéter fue retirado por sepsis generalizada. Concluyeron que los catéteres peritoneales tunelizados parecen ser un procedimiento seguro, efectivo y mínimamente invasivo para el tratamiento de la AM en pacientes oncológicos terminales.

Estos catéteres facilitan la evacuación de la ascitis en el domicilio de los pacientes, sin la necesidad de acudir a un centro de salud y evita punciones a repetición. Sin embargo, hace falta una mayor experiencia y estudios prospectivos aleatorizados.

Berchíd presentó el caso de un paciente con hepatocarcinoma en estadio terminal, al que se le realizó paracentesis evacuadora a nivel domiciliar, con buenos resultados en colaboración con el equipo de soporte de cuidados paliativos de la zona. El seguimiento domiciliar fue realizado por el médico de atención primaria.

La Food and Drug Administration (FDA) aprobó en 2005 el uso de los catéteres Pleur $X$ para el manejo de la $\mathrm{AM}^{(2)}$.

\section{Derivaciones (Shunt):}

No hay estudios comparativos exitosos entre los 2 tipos de "shunt". Tampoco hay estudios comparativos entre los diferentes tipos de catéteres y finalmente, no hay información exacta del grado de pérdida de proteínas que ocurre con el drenaje del catéter asociado a empeoramiento de los síntomas $^{(2)}$.

El pronóstico de vida que se solicita para colocar un shunt es, variando según los estudios, entre 1 y 3 meses de esperanza de vida $^{(16)}$ y se ha estimado que es efectivo en el control de síntomas en un $70 \%$ de los pacientes seleccionados ${ }^{(16)}$.

Ventajas: el ahorro de electrolitos, la retención de líquido ascítico rico en proteínas, se mantiene el balance hídrico del cuerpo y se evita drenajes externos, haciéndolo más aceptable para el paciente y su familiares.

Desventaja: riesgo de oclusión, edema pulmonar, trombosis venosa, mayor formación de seromas y coagulación intravascular diseminada.

Contraindicaciones para su realización: Ictericia (BT > $6 \mathrm{mg} / \mathrm{dl}$ ), coagulopatía, hipertensión portal y derrame pleural masivo. La insuficiencia cardiaca congestiva y la insuficiencia renal son contraindicaciones por la hemodilución y el exceso de volumen intravascular, producto del shunt $^{(2,3)}$. También, un contenido de proteínas mayor de $4 \mathrm{~g} / 1$ lo contraindica, debido a la pobre respuesta al shunt y las ascitis de origen gastrointestinal, por su pobre pronóstico ${ }^{(16)}$.

Las complicaciones son muy frecuentes, entre el 25 y $50 \%$ de los casos. Entre ellas la obstrucción (la más frecuente según Sangisetty), la coagulación vascular diseminada, el edema pulmonar, la diseminación del tumor, las infecciones y la presentación transitoria de fiebre ${ }^{(16)}$.

En relación a lo descrito anteriormente, la colocación de shunts no se considera un tratamiento apropiado y está contraindicado en pacientes con cáncer en fase terminal.

Se dice que la permeabilidad del shunt varia de 10 a 12 semanas ${ }^{(11)}$ sin embargo, para otros autores puede ir de 52 a 266 días $^{(1)}$. 


\section{CONCLUSIONES Y RECOMENDACIONES}

La ascitis maligna constituye una complicación importente en diversos tipos de tumores y genera importante morbilidad en los pacientes que la paceden.

Tiene múltiples causas $\mathrm{y}$, de acuerdo a sus características, puede ser clasificada en diversos grupos, con implicaciones fisiopatológicas y terapéuticas específicas, indispensables para dar un tratamiento apropiado.

En la actualidad se cuenta con diversas alternativas terapéuticas, que van a variar según las características propias del líquido ascítico y la sobrevida del paciente.

Siendo un padecimiento fundamentalmente de pacientes en etapa terminal, el reconocimiento y tratamiento oportuno y adecuado de la ascitis maligna tiene importante impacto en la calidad de vida de quienes la padecen.

\section{REFERENCIAS BIBLIOGRÁFICAS}

1. Saif M Siddiqui I Sohail M. Management of ascites due to gastrointestinal malignancy. Ann Saudi Med. 2009; 29(5):369-377.

2. Ammouri L Prommer E. Palliative treatment of malignant ascites: profile of catumaxomab. Biologics: targets \& therapy. 2010;4:103-110.

3. Guillén M Plancarte R Reyes D Guajardo J Chejne F. Manejo paliativo de la ascitis en pacientes oncológicos. Cancerología. 2008;3:149-157.

4. Berchid M Martínez V Ceacero C Medina J. Paracentesis evacuadora domiciliar en cuidados paliativos realizada por profesionales de atención primaria. Presentación de un caso. Semergen. 2013;39(3):161-164.

5. Eskander R Tewari K. Emerging treatment options for management of malignant ascites in patients with ovarian cancer. International Journal of Women's Health. 2012;4:395-404.

6. Word Health Organization. En nota descriptiva No 297 de Febrero del 2013. Revisada el 3 de Setiembre del 2013 en: http://www.who.int/mediacentre/factshe ets/fs297/es/
7. Becker G Galandi D Blum H. Malignant ascites: Systematic review and guideline of treatment. European Journal of Cancer. 2006;42:589-597.

8. Chung M Kozuch P. Treatment of malignant ascites. Current treatment options in oncology. 2008;9:215-233.

9. Rosenberg S. Palliation of malignant ascites. Gastroenterology clinics of North America. 2006;35:189-199.

10. Saiz R. Drenaje permanente tunelizado de la ascitis maligna: experiencia inicial con el catéter PleurX. Radiología. 2010;52(6):541-545.

11. Sangisetty S Miner T. Malignant ascites. A review of prognostic factors, pathophysiology and therapeutic measures. World $\mathrm{J}$ of gastrointestinal Surg. 2012;4(4):87-95.

12. Sales P Calsina-Berna A López M Cabrera M Poyato E Grimau I. Estudio descriptivo sobre el uso de catéteres peritoneales en el manejo de la ascitis maligna. Medicina Paliativa. 2012;19(1): 38-44.

13. Markman M. Palliation of symptomatic malignant ascites; an (often) unmet need. Oncology. 2012;82:313-314.

14. Cavazzoni E Bugiantella W Graziosi L Franceschini MS Donini A. Malignant ascites: pathophysiology and treatment. Int J Clin Oncol. 2013;18:1-9.

15. Hou W Sanyal A. Ascites: diagnosis and management. Med Clin N A. 2009; 93:801-817.

16. Salinas M Benítez MA González R. Diagnóstico y tratamiento de la ascitis en pacientes con cáncer en fase terminal. Medicina Paliativa. 2006;13(2);9499.

17. Mullard AP Bishop JM Jibani M. Intractable malignant ascites: an alternative management option. Journal of Palliative Medicine. 2011;14(2):251-253.

18. Plancarte R Guillén J Mayer F. Ascitis en los pacientes oncológicos. Fisiopatogenia y opciones de tratamiento. Revista de la sociedad española de dolor. 2004;11:156-162.

19. Coupe N Cox K Clark K Boyer M Stockler M. Outcomes of permanent peritoneal ports for the management of recurrent malignant ascites. Journal of Palliative Medicine. 2013;16(8):938940. 
20. Moses D. Tarascon. Palliative medicine pocketbook. 2012 p. 52.

DECLARACIÓN DE CONFLICTO DE INTERESES

Los autores declaran que no existen conflictos de intereses. 